CLINICAL STUDY

\title{
Number of autoantibodies and HLA genotype, more than high titers of glutamic acid decarboxylase autoantibodies, predict insulin dependence in latent autoimmune diabetes of adults
}

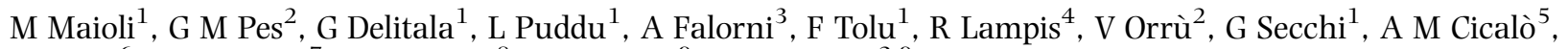 \\ R Floris $^{6}$, G F Madau ${ }^{7}, \mathrm{R}$ M Pilosu ${ }^{8}$, M Whalen ${ }^{9}$ and F Cucca ${ }^{2,9}$ \\ Departments of ${ }^{1}$ Internal Medicine and ${ }^{2}$ Biomedical Sciences, University of Sassari, I-07100 Sassari, Italy, ${ }^{3}$ Department of Internal Medicine, University \\ of Perugia, I-06126 Perugia, Italy, ${ }^{4}$ Department of Biomedical Sciences and Biotechnology, University of Cagliari, I-09121 Cagliari, Italy, ${ }^{5}$ Diabetes Unit, \\ ASL No. 3, I-08100 Nuoro, Italy, ' Diabetes Unit, Ospedale 'G. Brotzu', I-09121 Cagliari, Italy, ${ }^{7}$ Diabetes Unit, ASL No. 5, I-09170 Oristano, Italy, \\ ${ }^{8}$ Diabetes Unit, ASL No. 8, Ospedale 'S. Giovanni di Dio', I-09121 Cagliari, Italy and ${ }^{9}$ Institute of Neurogenetics and Neuropharmacology, CNR Cagliari, \\ I-09121 Cagliari, Italy \\ (Correspondence should be addressed to M Maioli; Email: marimaio@uniss.it)
}

\begin{abstract}
Objective: In latent autoimmune diabetes of adults (LADA), the progression into insulin-dependent diabetes is usually faster than in type 2 diabetes (T2D) but the factors influencing this progression are not completely known. In this study, we searched for sensitive markers associated with early development of insulin dependence.

Design: The screening of 5568 T2D patients for glutamic acid decarboxylase autoantibodies $(\mathrm{GAD} 65 \mathrm{Ab})$ identified $276 \mathrm{LADA}$ patients $(\mathrm{M}=131 ; \mathrm{F}=145)$ and in 251 of them, tyrosine phosphatase-2 (IA-2Ab) and thyroperoxidase autoantibodies (TPOAbs), some clinical features and genotype variation of the main type 1 diabetes (T1D) disease susceptibility loci (HLA-DRB1 and HLADQB1) were analyzed.

Results: Four years after the diagnosis of diabetes, high GAD65Ab titer was not significantly associated with faster progression toward insulin deficiency $(P=0.104)$. Patients with GAD65Ab and TPOAb or $\mathrm{IA}-2 \mathrm{Ab}$ or triple positivity for both islet and TPOAbs (GAD65Ab/IA-2Ab/TPOAb) showed a significantly faster disease progression $(P=0.002)$. Among 104 TPOAb-positive LADA patients, 10 received replacement therapy (L-thyroxine), 43 showed high TSH levels $(62.7 \%$ developed insulin dependence), and 3 had hyperthyroidism treated with methimazole. Multivariate analysis revealed a significant effect on disease progression only for TPOAb $(P=0.022)$, female gender $(P=0.036)$, low body mass index (BMI; $P=0.001$ ), and T1D high/intermediate risk HLA-DRB1/DQB1 genotypes grouped $(P=0.020)$.

Conclusions: High GAD65 Ab titers per se are not a major risk factor for disease progression in LADA, while the number of positive autoantibodies and HLA DRB1-DQB1 genotypes at high risk for T1D are significant predictors. Moreover, clinical characteristics such as low BMI and female gender are more likely to identify patients who will require insulin therapy within 4 years of diagnosis.
\end{abstract}

European Journal of Endocrinology 163 541-549

\section{Introduction}

About $5-10 \%$ of all patients diagnosed with type 2 diabetes (T2D) have markers of $\beta$-cells autoimmunity, such as glutamic acid decarboxylase (GAD65Ab) and tyrosine phosphatase-2 autoantibodies (IA-2Ab) (1-4). This form of 'autoimmune' diabetes, defined by Zimmet (5) as 'latent autoimmune diabetes of adults' (LADA), shows clinical features somewhat intermediate between classic early-onset type 1 diabetes (T1D) and T2D, and is characterized by the absence of ketoacidosis and insulin therapy for variable periods of time (6). The progressive destruction of $\beta$-cells is usually slower in LADA than in
T1D, and has been reported to be faster in the presence of high GAD65 Ab titers (7). The existence of distinct levels of GAD65 Ab in subgroups of LADA patients was recently highlighted in a large Italian study, which reported a clear bimodal distribution of GAD65Ab titers as well as the association of higher titers with a clinical phenotype more similar to T1D (8). Although this finding may suggest an intrinsic heterogeneity of LADA, no consistent data about a possible influence of GAD65 Ab titers on progression toward insulin dependence was provided in that study. Some authors agree that a high titer of GAD65Ab is associated with a shorter insulin-free period, whereas others do not 
support this view (9). However, the presence of multiple $\beta$-cell autoantibodies such as GAD65 Ab and islet cell antibodies (ICA) (10) or GAD65Ab and IA-2Ab (11) in adult patients diagnosed with T2D is highly correlated with a faster decline of islet function.

The potential of several factors such as C-peptide (12), age at diagnosis, and several lipid parameters (13) to predict progression toward insulin dependence has been investigated previously, but conflicting data have been reported so far due to the limited size of LADA cohorts analyzed and/or ethnic admixture (14). Inconsistent data has also been reported about the prevalence and impact of metabolic syndrome (MetS) in the clinical course of LADA (15). Besides this, some genetic markers, e.g. combinations of specific human leucocyte antigens (HLA) class II DRB1-DQB1 haplotypes and genotypes, have been found to be associated with the risk of progression in T1D. This raises the question whether variation at these loci also modulates the probability that a LADA patient will require insulin therapy sooner.

With the aim to inquire these LADA-related issues as well as to identify more sensitive markers associated with early development of insulin dependence - which may enable the physician to stratify the risk for insulin dependence in this form of diabetes - we retrospectively evaluated the clinical course during 4 years of observation of a large cohort of LADA patients from the ethnically homogeneous population of Sardinia (2).

\section{Patients and methods}

\section{Patients}

At the end of 2005, we started a multicentric study of T2D patients (age range 35-70 years) referred to five Diabetic Units of the island of Sardinia, Italy, to screen for the presence of pancreatic islet autoantibodies. Out of 5568 T2D patients, $276(4.9 \%)$ patients were GAD65 Ab positive, and 251 of them gave their consent to participate in the study, which was approved by the local ethics committee. The duration of diabetes in these patients dated back from $<8$ months to no longer than 5 years. None of the patients showed evidence of ketoacidosis and/or significant weight loss, nor had insulin therapy for at least 8 months from diagnosis. All patients were of Sardinian origin for at least two generations, and none of them were affected by severe liver and/or renal disease. Age and gender were recorded at diagnosis, whereas other clinical parameters including height, weight, body mass index (BMI), abdominal circumference, fasting glucose, creatinine, lipid profile (total and high-density lipoprotein (HDL) cholesterol, and triglycerides), uric acid, HbAlc, systolic, and diastolic blood pressure were collected from the clinical records provided by the referring Diabetic Units for a period between 12 and 18 weeks after the diagnosis of diabetes and the start of treatment to ensure adequate metabolic control. C-peptide was measured only in a limited number of patients whose sera had been available at diagnosis. All study participants were tested for MetS according to the International Diabetes Federation (IDF) worldwide consensus (16).

\section{Autoimmunity assessment}

All GAD65Ab-positive sera were also tested for IA-2Ab as well as other organ-specific autoantibodies such as anti-transglutaminase antibodies (tTGAbs) and antithyroperoxidase antibodies (TPOAbs). The results have been entered into ordinal variables taking into consideration single (only GAD65Ab), double (GAD65Ab + another autoantibody), or triple positivity (GAD65Ab+two additional autoantibodies). In 2510 GAD65 Ab-negative T2D patients, randomly selected from the 5568 T2D patients already screened, TPOAb and tTGAb were screened as well. TSH plasma level was measured by IRMA (TSH-US IRMA CT, Radim S.p.A., Rome, Italy) in all LADA patients as well as in a random sample of 251 GAD65Ab-negative T2D patients.

Both $\beta$-cell antibodies were measured using a radiobinding assay with in vitro-translated $\left[{ }^{35} \mathrm{~S}\right]$ methioninelabeled GAD65 or IA-2 (17). Results were expressed as an index defined as the ratio between the difference of counts per minute between sample and standard control (18). The \% intra-assay coefficient of variation of GAD65Ab and IA-2Ab for control samples designated at 35 anti-GAD65 units was 5.7 and $10 \%$ respectively (3). All GAD65 Ab results were double checked by an independent laboratory (University of Perugia, Italy), using an RIA that uses ${ }^{125}$ I-radiolabeled human GAD65 (RSR Ltd, Cardiff, UK), with a $100 \%$ concordance of positive/negative results and a high correlation of antibody titers between the two assays $(r=0.936 ; P=0.03)$. tTGAb and TPOAb were tested by RIA using commercial kits (Medipan, Berlin, Germany). During the insulin-free period, most patients were treated with nutritional therapy and, when necessary, with hypoglycemic agents. The progression toward $\beta$-cell failure was estimated through time (in months) between diagnosis and the start of the insulin therapy as soon as patients showed postprandial glucose levels above $180 \mathrm{mg} / \mathrm{dl}$ despite maximum allowed dose of hypoglycemic drugs, significant body weight loss in spite of the consumption of the usual diet, or the appearance of ketosis.

\section{HLA genotyping}

HLA class II genotyping of the HLA-DRB1, DQA1, and DQB1 genes was performed by dot-blot analysis with sequence-specific oligonucleotide probes as previously reported (19). The HLA class II genotypes were ranked as high, intermediate, or low risk for T1D (19). The high-risk category included persons with two highrisk haplotypes; the intermediate risk category was 
constituted mainly by individuals carrying genotypes given by the combinations of one high risk and one permissive-neutral haplotype while the low risk category encompassed genotypes constituted by two copies of negatively associated haplotypes or combinations of negatively associated and neutral haplotypes.

\section{Statistical analysis}

SPSS statistical software (version 16.0, Chicago, IL, USA) was used to perform most analyses. Power analysis was performed with EpiCalc 2000 version 1.02. Results were expressed as means \pm s.D. or frequencies. Statistical differences between groups of quantitative variables were tested using nonparametric Mann-Whitney $U$ test. Differences in frequencies were tested by the $\chi^{2}$ test with Yates' correction or exact Fisher test, whenever appropriate. Patients were divided into two groups according to the GAD65Ab mode titers (high- or low anti-GAD65Ab titers based on a cut-off index 0.5 corresponding to the sample median). Receiving operating curve (ROC) analysis was also performed to evaluate the overall performance of GAD65Ab titer in discriminating patients who required insulin therapy and to find an appropriate cut-off. Kaplan-Meier (KM) curves were plotted, and log-rank test was performed to identify possible markers capable to influence the progression into insulin dependence. A multivariate Cox proportional hazard model was also constructed and, apart from gender and age at diagnosis, other variables such as number and titer of autoantibodies, and the HLA-associated genetic risk, were included in the model. Hazard ratios (HRs) were computed, and the log-likelihood ratio test was used for model testing.

\section{Results}

\section{Autoantibodies and clinical parameters in patients and controls}

The screening for GAD65Ab in $5568 \mathrm{~T} 2 \mathrm{D}$ patients diagnosed between mid-2000 and the end of 2005 revealed the presence of $276 \mathrm{GAD} 65 \mathrm{Ab}$-positive subjects $(4.9 \%)$. The number of males and females were $131(47.4 \%)$ and 145 (52.6\%) respectively, with a F/M ratio of 1.1. Among GAD65Ab-negative subjects, the number of males and females were 1160 (46\%) and $1350(54 \%)$ respectively. In total, 251 patients out of 276 gave their informed consent for further investigation. The patients who refused to participate in the study showed antibody titers and phenotypic characteristics very similar to those who eventually continued the study. The clinical characteristics of patients who participated in the study, subdivided by GAD65Ab titers, are reported in Table 1 compared with those of 2510 GAD65Ab-negative T2D patients. Most of the clinical parameters in the LADA cohort as a whole showed average values distinctly different from T2D, especially BMI, waist circumference, blood pressure, the frequency of MetS, and TPOAb positivity. The distribution of GAD65Ab titers among all LADA patients showed two modes with a peak around the log-index value of -2.0 and another around +1.0 respectively (Fig. 1). The number of patients with high (index $\geq 0.5$ ) and low (index <0.5) GAD65Ab titer was $127(51 \%)$ and 124 (49\%) respectively; 53 patients exhibited also positivity for IA-2Ab (21\% of the total) and 104 (41\%) were positive for TPOAb. Only one female patient resulted positive for serum tTGAb $(0.4 \%)$. The comparison of phenotypic characteristics between high and low GAD65Ab titer groups in LADA patients showed

Table 1 Clinical and laboratory variables (mean \pm s.D.) in latent autoimmune diabetes of adults (LADA) patients (subdivided according to GAD65Ab titer) and GAD65-negative type 2 diabetes (T2D) controls.

\begin{tabular}{|c|c|c|c|c|}
\hline Variables & Low titer GAD65Ab & High titer GAD65Ab & LADA & GAD65Ab negative T2D \\
\hline Number of cases (\%) & $124(49)$ & $127(51)$ & 251 & 2510 \\
\hline Mean age at diagnosis (years) & $55.4 \pm 11.7$ & $53.4 \pm 10.9$ & $54.3 \pm 11.2$ & $57.7 \pm 10.1^{\dagger}$ \\
\hline Mean age at recruitment (years) & $56.0 \pm 12.8$ & $54.5 \pm 10.4$ & $55.2 \pm 11.6$ & $58.1 \pm 11.9$ \\
\hline Gender (M:F) & $59: 65$ & $58: 69^{\ddagger}$ & 117:134 & $1160: 1350^{\ddagger}$ \\
\hline Body mass index $\left(\mathrm{kg} / \mathrm{m}^{2}\right)$ & $28.8 \pm 4.9$ & $26.9 \pm 4.6^{\star}$ & $27.7 \pm 4.8$ & $30.8 \pm 6.1^{\dagger}$ \\
\hline Waist circumference $(\mathrm{cm})$ & $97.5 \pm 11.8$ & $94.9 \pm 10.1$ & $96.0 \pm 10.8$ & $101.1 \pm 12.1^{\dagger}$ \\
\hline Total cholesterol (mg/dl) & $206.9 \pm 44.5$ & $213.5 \pm 43.8$ & $210.9 \pm 44.0$ & $220.9 \pm 54.4^{\dagger}$ \\
\hline HDL cholesterol (mg/dl) & $55.1 \pm 14.0$ & $55.8 \pm 18.1$ & $55.5 \pm 16.5$ & $48.3 \pm 21.4^{\dagger}$ \\
\hline Triglycerides (mg/dl) & $118.2 \pm 76.0$ & $117.6 \pm 65.1$ & $117.7 \pm 69.3$ & $123.2 \pm 108.2$ \\
\hline Fasting glucose (mg/dl) & $134.3 \pm 28.1$ & $136.8 \pm 31.4$ & $135.2 \pm 29.5^{\star}$ & $138.8 \pm 27.4$ \\
\hline $\mathrm{HbA1c}(\%)$ & $7.3 \pm 2.7$ & $6.5 \pm 1.9$ & $6.9 \pm 2.4^{\star}$ & $7.1 \pm 1.8$ \\
\hline Uric acid (mg/dl) & $5.2 \pm 3.3$ & $4.7 \pm 1.7$ & $4.9 \pm 2.1^{*}$ & $5.3 \pm 2.8$ \\
\hline Metabolic syndrome (\%) & $59(47)$ & $47(\overline{37})^{\ddagger}$ & $106(42)$ & $2184(87)^{\S}$ \\
\hline Systolic blood pressure (mmHg) & $135.3 \pm 20.4$ & $133.8 \pm 18.8$ & $134.3 \pm 19.4$ & $141.4 \pm 7.9^{\dagger}$ \\
\hline Diastolic blood pressure $(\mathrm{mmHg})$ & $82.3 \pm 10.9$ & $81.3 \pm 9.6$ & $81.7 \pm 10.1$ & $87.3 \pm 12.6^{\dagger}$ \\
\hline TPOAb positive (\%) & $42(34)$ & $62(49)^{\|}$ & $104(41)$ & $290(11)^{\S}$ \\
\hline
\end{tabular}

${ }^{*}$ High versus low GAD65Ab titer $\left(P=0.008\right.$, Mann-Whitney $U$ test). ${ }^{\dagger}$ LADA versus GAD65Ab-negative T2D $\left(P<0.001\right.$, Mann-Whitney $U$ test). ${ }^{\ddagger}$ NS (Fisher's exact test). ${ }^{\S} P<0.0001$ (Fisher's exact test). " $P=0.020$ (Fisher's exact test). 


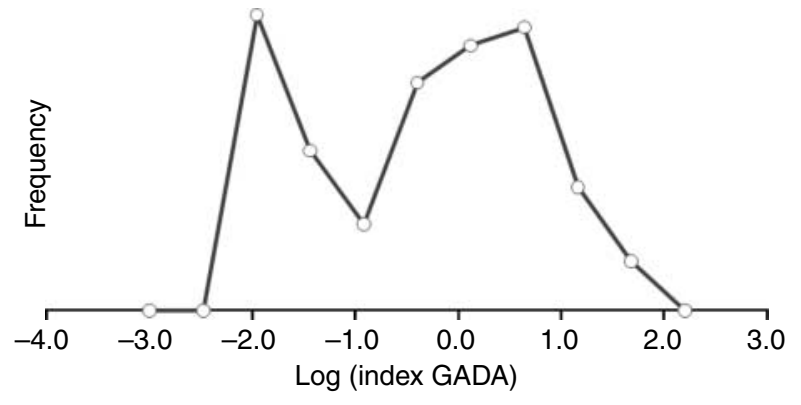

Figure 1 Bimodal distribution of $\log _{\mathrm{e}}$ (GAD65Ab index) among 251 LADA patients.

a significant difference only in BMI $(P=0.008)$ and in the frequency of TPOAb positivity $(P=0.001$; Table 1$)$.

Among the 104 TPOAb-positive LADA patients, ten received replacement therapy (L-thyroxine, $\mathrm{L}-\mathrm{T}_{4}$ ), and three patients were diagnosed with hyperthyroidism requiring methimazole treatment. In other 43 patients, TSH values above the cut-off limit of $4.0 \mathrm{mU} / \mathrm{l}$ were detected $(41.3 \%)$, whereas this percentage was as low as $4.5 \%$ in GAD65Ab-negative T2D patients. These patients had mild biochemical hypothyroidism, and did not receive $\mathrm{L}-\mathrm{T}_{4}$ treatment.

\section{Progression to insulin dependence}

The proportion of LADA patients who required insulin therapy within 4 years of diagnosis was $37.4 \%$. Table 2 shows the phenotype of LADA patients according to their progression into insulin dependence. HDL cholesterol levels and the frequency of TPOAb positivity were significantly higher, whereas BMI and waist circumference were significantly lower in patients who progressed into insulin dependence. Four out of ten LADA patients who were treated with substitutive hormonal therapy, two out of three patients with hyperthyroidism, and 27 out of $43(62.7 \%)$ with high TSH levels developed insulin dependence within 4 years. Moreover, 16 LADA patients out of $61(26 \%)$ with TSH levels $<4.0 \mathrm{mU} / \mathrm{l}$ progressed toward insulin dependence. All other variables were similar in both groups.

The KM curves for LADA patients subdivided according to GAD65 Ab mode titers adjusted for age and sex are represented in Fig. 2a where no significant difference (log-rank test: $P=0.104$ ) was observed. Figure $2 b$ and $c$ show KM curve for patients subdivided by IA- $2 \mathrm{Ab}$ and TPOAb positivity revealing a significant association between double antibody positivity and a faster insulin dependence (GAD65 $\mathrm{Ab}+/ \mathrm{IA}-2 \mathrm{Ab}+$ ) (log-rank test; $P=0.020)$ or $(\mathrm{GAD} 65 \mathrm{Ab}+/ \mathrm{TPOAb}+)$ (log-rank test: $P=0.007)$. Interestingly, regardless of GAD65 Ab titers, LADA patients with triple positivity for both islet and thyroid antibodies (GAD65Ab/IA-2Ab/ $\mathrm{TPOAb}$ ) significantly showed faster disease progression $(P=0.001)$.
As reported in Fig. 3a and b, KM analysis showed significant association (log-rank test: $P=0.001$ ) between double antibody positivity (GAD65 Ab + / $\mathrm{TPOAb}+$ ) with a faster insulin dependence in IA-2Abnegative patients, whereas the association of double antibody positivity $(\mathrm{GAD} 65 \mathrm{Ab}+/ \mathrm{IA}-2 \mathrm{Ab}+)$ was not significant in TPOAb-negative patients.

HLA-DRB1 and HLA-DQB1 genotypes that confer either high or intermediate risk to T1D were overrepresented among LADA patients who required insulin therapy 4 years or less following diagnosis $(P=0.036$, Fisher's exact test; Table 2), and KM analysis showed association with progression of diabetes (log-rank test; $P=0.013$; Fig. 4).

Cox regression analysis (Table 3 ) showed that the four most relevant factors for future disease progression were a low BMI $(\mathrm{HR}=0.853 ; P=0.001)$, HLA-associated genetic risk (1.473; $P=0.020)$, TPOAb $(\mathrm{HR}=2.018$; $P=0.022)$, and female gender $(\mathrm{HR}=0.520 ; P=0.036)$. On the contrary, IA-2Ab $(\mathrm{HR}=1.491 ; P=0.236)$ positivity and age at diagnosis $(\mathrm{HR}=0.981$; $P=0.209$ ) were not significantly associated. Finally, C-peptide levels measured in a limited number of LADA patients at diagnosis of diabetes did not differ between the two groups and did not influence the progression of diabetes (data not shown). A simple predictive score was constructed on the basis of these four variables giving one point for being a female and additional points for having a BMI in the lower tertile, a positivity for TPOAb

Table 2 Clinical and laboratory variables in latent autoimmune diabetes of adults patients (subdivided according to the progression into insulin dependence within 4 years).

\begin{tabular}{|c|c|c|c|}
\hline Variables & $\begin{array}{l}\text { Insulin } \\
\text { independent }\end{array}$ & $\begin{array}{l}\text { Insulin } \\
\text { dependent }\end{array}$ & $P$ value \\
\hline Number of cases (\%) & $157(63)$ & $94(37)$ & \\
\hline $\begin{array}{l}\text { Mean age at diagnosis } \\
\text { (years) }\end{array}$ & $54.6 \pm 10.6$ & $54.1 \pm 11.4$ & $\mathrm{NS}^{\mathrm{a}}$ \\
\hline Gender (M:F) & $81: 76$ & $36: 58$ & $0.049^{b}$ \\
\hline $\begin{array}{l}\text { Fasting plasma } \\
\text { glucose (mg/dl) }\end{array}$ & $135.2 \pm 29.5$ & $138.8 \pm 27.4$ & NS \\
\hline $\mathrm{HbA} 1 \mathrm{c}(\%)$ & $6.9 \pm 2.4$ & $7.1 \pm 1.8$ & NS \\
\hline $\begin{array}{l}\text { Body mass index } \\
\left(\mathrm{kg} / \mathrm{m}^{2}\right)\end{array}$ & $29.1 \pm 5.4$ & $25.8 \pm 3.6$ & $0.0001^{\mathrm{a}}$ \\
\hline $\begin{array}{l}\text { Waist circumference } \\
(\mathrm{cm})\end{array}$ & $98.5 \pm 9.0$ & $91.4 \pm 12.8$ & $0.032^{\mathrm{a}}$ \\
\hline $\begin{array}{l}\text { Total cholesterol } \\
\text { (mg/dl) }\end{array}$ & $210.7 \pm 47.9$ & $209.2 \pm 39.8$ & NS \\
\hline $\begin{array}{l}\text { HDL cholesterol } \\
(\mathrm{mg} / \mathrm{dl})\end{array}$ & $52.0 \pm 12.2$ & $57.6 \pm 14.9$ & $0.015^{\mathrm{a}}$ \\
\hline Triglycerides (mg/dl) & $117.7 \pm 53.4$ & $112.9 \pm 85.3$ & NS \\
\hline MetS (\%) & $70(44)$ & $36(38)$ & NS \\
\hline $\begin{array}{l}\text { Systolic blood } \\
\text { pressure (mmHg) }\end{array}$ & $134.4 \pm 20.8$ & $132.8 \pm 16.9$ & NS \\
\hline $\begin{array}{l}\text { Diastolic blood } \\
\text { pressure }(\mathrm{mmHg})\end{array}$ & $82.7 \pm 10.5$ & $80.4 \pm 9.4$ & NS \\
\hline TPO positivity (\%) & $55(35)$ & $49(52)$ & $0.008^{b}$ \\
\hline HLA-related risk & $66(42)$ & $53(56)$ & $0.036^{\mathrm{b}}$ \\
\hline
\end{tabular}

${ }^{a}$ Mann-Whitney $U$ test.

${ }^{\mathrm{b}}$ Fisher's exact test. 


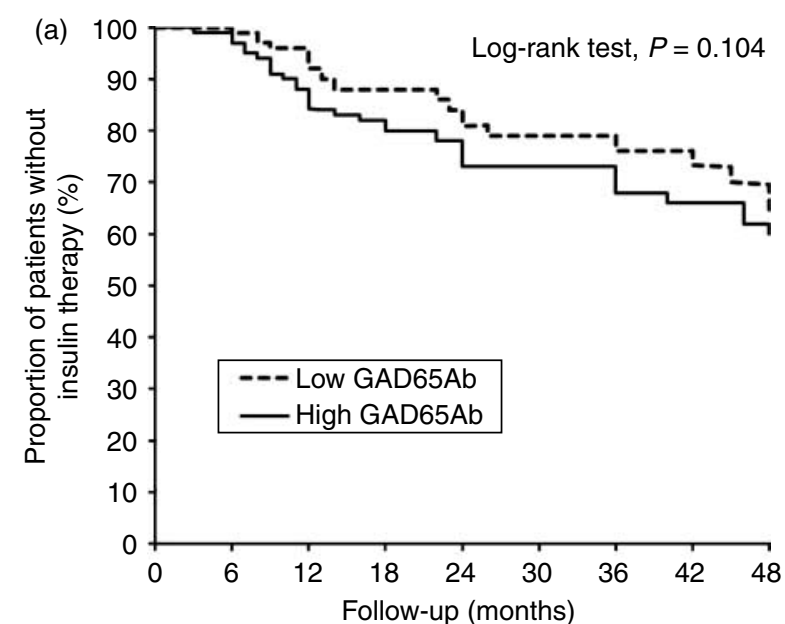

This score did not correlate significantly with the GADA levels $(r=0.011 ; P=0.204)$.

Furthermore, we observed that the percentage of sulfonyl urea-treated LADA patients in 'insulindependent' and 'noninsulin-dependent' subgroups were comparable ( 28 vs $26 \%$; NS). Besides, markers of metabolic controls, such as fasting glucose and $\mathrm{HbA1c}$, were not significantly different in either groups (Table 2).

Finally, the proportion of LADA patients who met the criteria for MetS was $42 \%$ in the whole cohort, while in the low and high GAD65 Ab groups were 47 and $37 \%$ respectively. A tendency of a lower proportion of patients with MetS was found among those who required insulin early, although the rate of progression did not differ between patients with or without MetS according to KM analysis (log-rank test; $P=0.209$ ). Actually, a significantly increased waist circumference was observed in patients who did not progress into insulin dependence (Table 2).
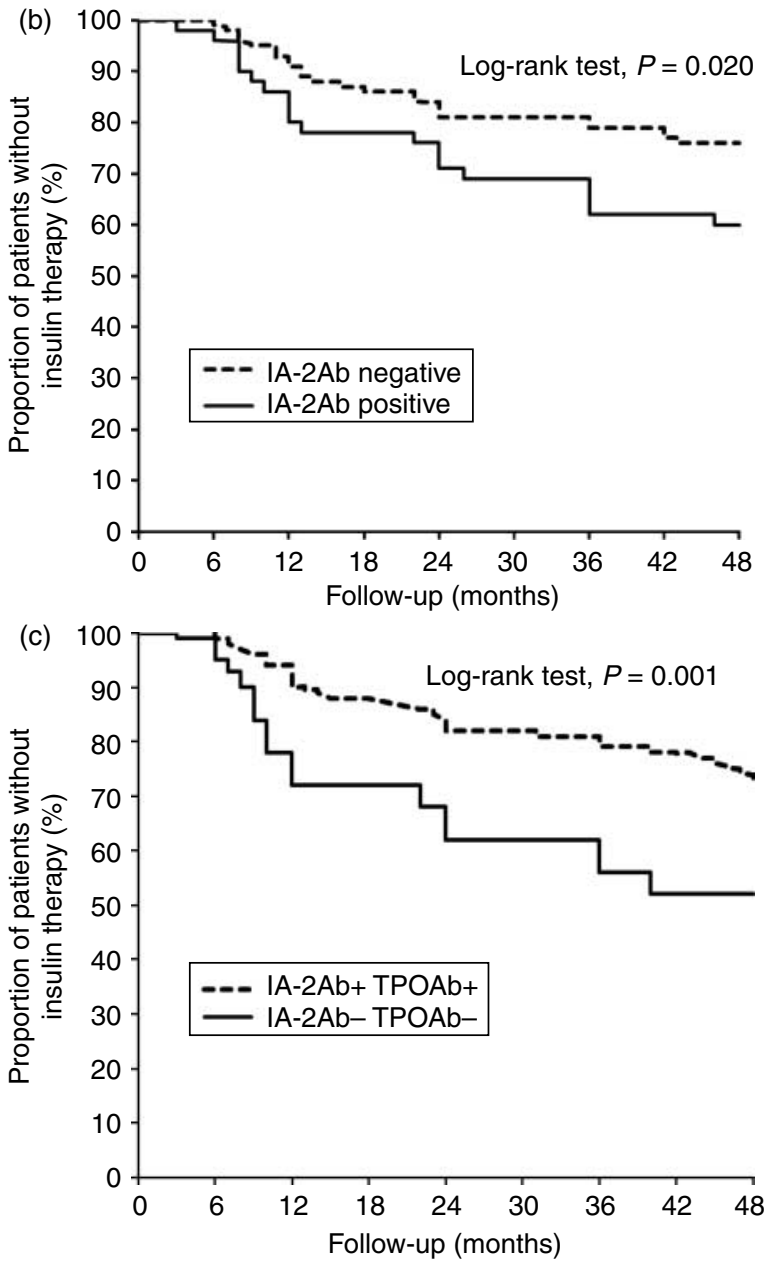

Figure 2 Kaplan-Meier curve showing the effect of the GAD65Ab titer (a), IA-2Ab (b), and TPOAb (c) for the subsequent development of insulin dependence in LADA cohort.

and a 'high risk' HLA genotype. Half of patients scored $<2$, and the other half 2 . This scoring system was highly predictive for the subsequent development of insulin deficiency (log-rank test, $P=0.00019$ ).

\section{Discussion}

In our cross-sectional study, we observed a clear bimodal distribution of GAD65 Ab values in agreement with the data reported previously (8). Analysing the clinical characteristics in the 'high titer' versus 'low titer' GAD65 Ab subgroups, we found the existence of a modest phenotypic heterogeneity since only BMI displayed a significant difference between the two groups, while triglyceride, total and HDL cholesterol levels, and blood pressure were not different, in contrast with what previously reported (13). On the other hand, a wide difference was found between LADA patients as a whole and GAD65 Ab(-) T2D patients, as reported by several studies $(20,21)$. An interesting question is whether high GAD65Ab titers are also capable to predict $\beta$-cell exhaustion.

In our study, we analyzed the predictive value of GAD65Ab titers in relation to the progression of the disease into insulin dependence taking advantage of careful clinical observations spanning 4 years. The choice of a 4-year period is due to the need to evaluate the mid-term evolution of the ongoing destructive process against $\beta$-cells. Our data revealed a slight but not significant association of GAD65 Ab titer with faster progression of diabetes toward insulin deficiency. However, given the limited statistical power of our sample size, this data must be taken cautiously. In our comparisons between high and low GAD65Ab titer groups, as far as the disease progression is concerned, we had only $9 \%$ points and a proportion of about $50 \%$, so that the statistical power was $40 \%$ for a one-sided test. The poor predictive value of high GAD65Ab levels into disease progression also persisted when several cutoff values were tested. In fact, although ROC analysis identified better discriminating values of cut-offs $\geq 0.5$, Cox analysis failed to reveal any significant 

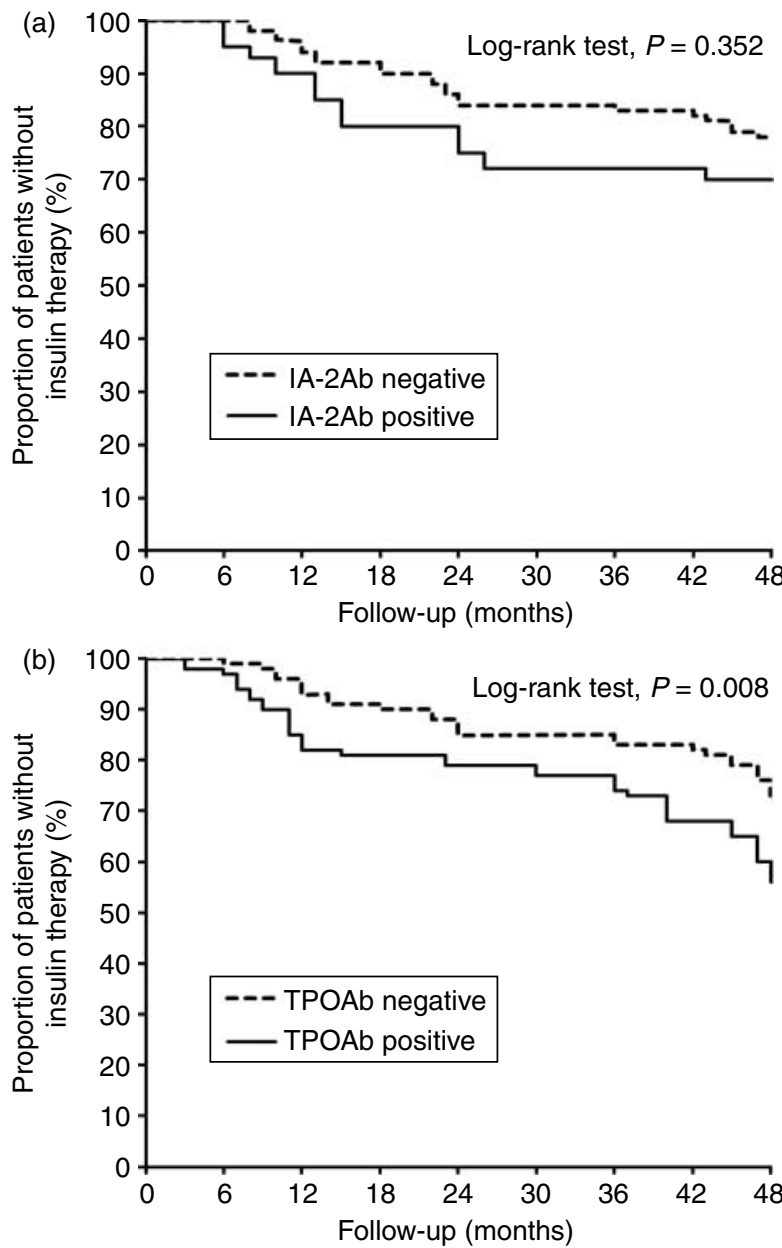

Figure 3 Kaplan-Meier curve showing the effect of IA-2Ab in TPOAb negative (a) and of TPOAb in IA-2Ab negative (b) for the subsequent development of insulin dependence in LADA cohort.

improvement in predictive value of GAD65 $\mathrm{Ab}$ titer when all other confounding variables were included in the model (data not shown).

Several authors reported a strong predictive power of high GAD65 Ab levels in the progression of LADA toward insulin dependence $(11,22)$. However, in these studies, regression analysis was not performed to control potential confounding variables. Where such analysis was carried out in large cohorts (9), the predictive role of high GAD65 Ab levels resulted to be negligible. Furthermore, some studies which reported a positive association between high GAD65 $\mathrm{Ab}$ titer and progression even after multivariate analysis $(7,10)$ did not take into account HLA-associated T1D susceptibility markers. Thus, the lack of control for confounders may have produced a spurious association between GAD65Ab titers and disease progression. Higher GAD65Ab levels were reported in patients with the age range of 7-16 years presenting with typical type 1 diabetes (23) carrying HLA-DR3 haplotype in comparison with subjects carrying DR4 haplotypes. Furthermore,
Buzzetti et al. (8) showed an increased frequency of HLA-DR3 subjects in high GAD65Ab LADA subgroup. Similarly, in our LADA cohort, HLA-DR 3 carriers showed a significantly increased GAD65 Ab level in comparison with carriers of HLA-DR4 and other less frequent haplotypes. This suggests that at least in Caucasian populations, subgroups of patients with an increased frequency of HLA-DR 3 may have elevated serum level of GAD $65 \mathrm{Ab}$ regardless of the severity of the underlying autoimmune process and clinical expression of the disease. Accordingly, in our cohort, the frequency of HLA-DR 3 carriers was slightly higher in high GAD65 Ab subgroup, whereas the overall distribution of HLA susceptibility genotypes did not differ. A study of Kasuga et al. (24) in 43 Japanese LADA patients reported both higher GAD65Ab levels and an increased frequency of HLA-DR4 in patients who progressed faster toward insulin dependency. Unfortunately, these findings, which seem to support a role for genetic background on the evolution of the disease, have been obtained in a non-Caucasian ethnic group that shows both HLA Class II haplotype distribution and genetic associations with autoimmune diabetes very different than those of a Sardinian population, and are not easily comparable with our data.

The other predictive markers of progression into insulin dependence that we have found do not suffer from statistical limitations.

In GAD65Ab-positive patients often other organspecific markers of autoimmunity such as IA-2Ab and anti-thyroid antibodies are present (25). Our data indicate that irrespective of the level of GAD65Ab titer, the occurrence of IA-2Ab and/or TPOAb is associated with a significantly increased evolution of the disease compared with GAD65Ab-positive patients

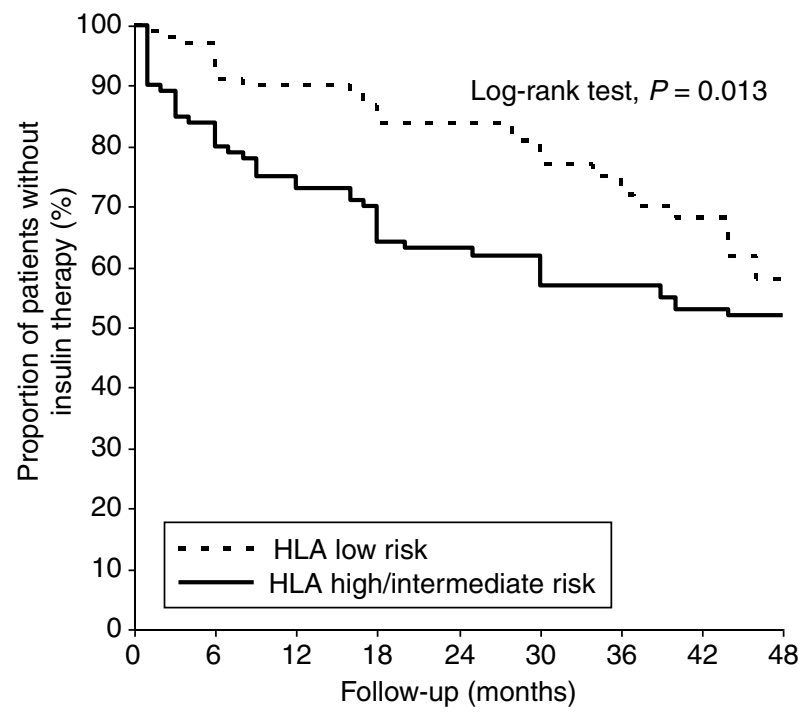

Figure 4 Kaplan-Meier curve showing the effect of HLA-associated genetic risk for the subsequent development of insulin dependence in LADA cohort. 
Table 3 (a) Univariate statistics using Cox's proportional hazard model, for the variables predicting insulin deficiency within 4 years in 251 latent autoimmune diabetes of adults patients. (b) The results of Cox's proportional hazard model using forward selection on the basis of the likelihood ratio statistic for the test group.

\begin{tabular}{lrccc}
\hline Variable & \multicolumn{1}{c}{$\begin{array}{c}\text { 95\% Confidence } \\
\text { interval for HR }\end{array}$} & Significance \\
\hline $\begin{array}{l}\text { (a) } \\
\text { Age at } \\
\text { diagnosis }\end{array}$ & -0.018 & 0.982 & $0.953-1.011$ & 0.225 \\
$\quad$ Gender & -0.695 & 0.499 & $0.269-0.928$ & 0.028 \\
GAD65Ab & -0.526 & 0.591 & $0.307-1.138$ & 0.115 \\
IA-2Ab & 0.403 & 1.497 & $0.772-2.900$ & 0.232 \\
TPOAb & 0.861 & 2.366 & $1.200-4.665$ & 0.013 \\
BMl & -0.166 & 0.847 & $0.778-0.922$ & 0.001 \\
HLA risk & 0.366 & 1.442 & $1.029-2.021$ & 0.034 \\
(b) & & & & \\
Gender & -0.653 & 0.520 & $0.283-0.957$ & 0.036 \\
TPOAb & 0.702 & 2.018 & $1.104-3.686$ & 0.022 \\
BMl & -0.159 & 0.853 & $0.786-0.925$ & 0.001 \\
HLA risk & 0.387 & 1.473 & $1.064-2.040$ & 0.020 \\
\hline
\end{tabular}

with no further autoimmune markers. Therefore, we confirm the results of previous studies, which showed that GAD65 Ab together with an additional specific anti$\beta$-cell antibody (ICA) in the same patient is associated with a poorer long-term prognosis, a feature which was reported in relatives of T1D patients (26). This is consistent with the concept that apart from GAD65Ab, the occurrence of both IA-2 Ab and TPOAb seem to identify subgroups of LADA patients who will develop insulin deficiency very soon.

Actually, the effect of IA-2Ab seems to be modulated by the copresence of TPOAb, in fact when only $\mathrm{IA}-2 \mathrm{Ab}+\mathrm{TPOAb}-$ patients are considered, the effect on progression is no longer apparent. On the contrary, the predictive value of TPOAb on disease progression is significantly high even in IA-2Ab-negative patients, suggesting a more powerful predictive effect of antithyroid antibodies on diabetes evolution. Accordingly, when IA-2Ab and TPOAb have been entered in the Cox model, only TPOAb showed a significant effect on disease progression.

Our findings are in line with data recently reported in a Japanese study, although in a very limited number of LADA patients, about an association between the presence of TPOAb and $\beta$-cell failure (27). Although at the moment we do not have any definite explanation of this finding, a possibility that should be considered is that the presence of extrapancreatic autoimmunity markers at diagnosis may reflect a more extended underlying immune process against $\beta$-cells. Clearly, further investigations in larger LADA cohorts are necessary to better understand the role of TPOAb in disease progression.

A substantial proportion of LADA patients with antithyroid antibodies also displayed a high frequency of subclinical or clinical thyroid disease. Four out of ten patients who were treated with substitutive hormonal therapy $\left(\mathrm{L}-\mathrm{T}_{4}\right)$, two out of three patients affected by hyperthyroidism, and $62.7 \%$ of patients with high TSH levels progressed rapidly into $\beta$-cell failure. Additionally, 16 patients with TPOAb positivity and low TSH progressed to insulin dependence. These data indicate that LADA patients should be routinely screened for thyroid autoimmunity as they have an increased risk of clinical thyroid disease as well as to progress faster into $\beta$-cell exhaustion. On the contrary, the frequency of positivity for markers of celiac disease was not different from the GAD65Ab-negative population.

Furthermore, on the basis of our multivariate analysis, the frequency of HLA DRB1-DQB1 genotypes that are most strongly associated with T1D risk is also associated with higher risk of insulin requirement within 4 years of diagnosis, and this effect remained even after adjustment of the other variables.

Our data also showed that another marker of disease progression is gender. In fact, although the $\mathrm{F} / \mathrm{M}$ ratio in LADA patients at diagnosis was close to 1 , in line with other reports (28), the evolution of the disease into insulin dependence in our cohort was faster in women. Therefore, we think it may be linked to a different body composition, i.e. a lower muscle mass and increased insulin resistance in women compared with men. These findings are similar to the gender-dependent progression to $\beta$-cell failure reported by Lindholm et al. (29) in GAD65Ab-positive patients in the age range of 40-59 years requiring insulin during the first year of diabetes as well as by Turner et al. (6) in diabetes subjects older than 45 years in the UKPDS cohort. Another independent factor associated with progression of diabetes is a low BMI, again in agreement with previous studies $(6,12,13)$. Multivariate analysis showed that its predictive value persisted even after adjustment for all the other variables. In fact, when the full model using a forward stepwise procedure was run, BMI, gender, TPOAb, and HLA genetic risk were all highly significant. Conversely, age at diagnosis, IA-2Ab, and GAD65Ab titer did not appear in the final regression model, and therefore, they cannot be considered independent predictors of progression. Despite higher HDL cholesterol levels in patients who progressed into insulin dependence, it did not prove to be a significant marker of progression in the multivariate analysis. We also evaluated the possibility that sulfonylurea treatment, before starting insulin therapy, could have accelerated disease progression, thus acting as a confounder. Actually, the percentage of LADA patients treated with sulphonylurea, in the two groups with and without $\beta$-cell failure, was comparable ( 26 vs $28 \%$; NS). Therefore, it is unlikely that sulphonylurea treatment may have influenced disease progression in our cohort.

In addition, we do not think that disease progression may have been differentially influenced by glucotoxicity as no significant difference in fasting glucose and $\mathrm{HbA1c}$ levels was noticed between insulin-dependent and noninsulin-dependent LADA patients.

Finally, in our study, we addressed also the problem of the influence of MetS on disease evolution, as this 
condition is supposed to affect negatively the course of diabetes. In our cohort, $42 \%$ of LADA patients had MetS according to IDF criteria, and its frequency was not significantly different between low and high GAD65 Ab group. However, its frequency was increased, although not significantly, in patients who did not require insulin therapy. In the paper by Buzzetti et al. (8) which adopted the ATPIII criteria instead of IDF to diagnose MetS, the frequency of MetS in LADA patients with low GADA titer was significantly higher in comparison with our patients. The high frequency MetS in that study (8) could be related to the high frequency of hypertriglyceridemia, which was virtually absent in our patients. This might be attributed to a better metabolic control of our patients, as reflected by lower fasting glucose and HbA1c levels. Other authors (9) also reported a MetS frequency much closer to that found in our study.

Interestingly, a significant lower frequency of insulin dependence was observed in patients with higher waist circumference, which is the essential component of MetS according to the IDF criteria. This is probably due to the fact that in patients with the same level of $\beta$-cell impairment, insulin resistance associated with MetS was able to increase fasting glucose levels earlier than in patients who did not have it. Therefore, LADA patients who presented with MetS at the time of recruitment probably had a residual islet function better than patients without MetS, although it cannot be ruled out that an excess of fat mass may play a protective role on the autoimmune process itself. Accordingly, evidence from NOD mice with leptin receptor knockout causing hyperleptinemia showed that obesity may delay the onset of insulin dependence (30).

In conclusion, the markers of progression analyzed in this study can help to improve the definition of the risk of LADA patients developing insulin dependence. However, as they explain only $42 \%$ of the variability of the insulin-free period, it is advisable to find additional markers to help the physician decide who should be treated appropriately with insulin therapy in LADA.

\section{Declaration of interest}

The authors declare that there is no conflict of interest that could be perceived as prejudicing the impartiality of the research reported.

\section{Funding}

This work was done using research grant from MIUR (Italian Ministry for University and Research) (PRIN 2007) (scientific coordinator: Prof. M Maioli) and partly done using grant no. 478 from Regione Autonoma della Sardegna (Diabetes Mellitus and autoimmune diseases) (scientific coordinator: Prof. G Delitala).

\section{References}

1 Irvine WJ, McCallum CJ, Gray RS, Campbell CJ, Duncan LJ, Farquhar JW, Vaughan H \& Morris PJ. Pancreatic islet-cell antibodies in diabetes mellitus correlated with the duration and type of diabetes, coexistent autoimmune disease, and HLA type. Diabetes 197726 138-147. (doi:10.2337/diabetes.26.2.138)
2 Maioli M, Alejandro E, Tonolo G, Gilliam LK, Bekris L, Hampe CS, Obinu DA, Manconi A, Puddu L, Lynch K, Lernmark A \& Study Group for the Genetics of Diabetes in Sardinia. Epitope-restricted 65-kilodalton glutamic acid decarboxylase autoantibodies among new-onset Sardinian type 2 diabetes patients define phenotypes of autoimmune diabetes. Journal of Clinical Endocrinology and Metabolism 200489 5675-5682. (doi:10.1210/jc.2004-0864)

3 Maioli M, Tonolo G, Bekris L, Cirillo R, Schranz D, Cossu E, Ciccarese M, Lernmark A \& Study Group for the Genetics of Diabetes in Sardinia (SGGDS). GAD65 and IA-2 autoantibodies are common in a subset of siblings of Sardinian type 2 diabetes families. Diabetes Research and Clinical Practice 200256 41-47. (doi:10.1016/S0168-8227(01)00348-5)

4 Pozzilli P \& Di Mario U. Autoimmune diabetes not requiring insulin at diagnosis (latent autoimmune diabetes of the adult): definition, characterization, and potential prevention. Diabetes Care 200124 1460-1467. (doi:10.2337/diacare.24.8.1460)

5 Zimmet P. The pathogenesis and prevention of diabetes in adults: genes, autoimmunity and demography. Diabetes Care 199518 1050-1064.

6 Turner R, Stratton I, Horton V, Manley S, Zimmet P, Mackay IR, Shattock M, Bottazzo GF \& Holman R. UKPDS 25: autoantibodies to islet-cell cytoplasm and glutamic acid decarboxylase for prediction of insulin requirement in type 2 diabetes. UK Prospective Diabetes Study Group. Lancet $1997 \mathbf{3 5 0}$ 1288-1293. (doi:10.1016/S0140-6736(97)03062-6)

7 Genovese S, Bazzigaluppi E, Gonçalves D, Ciucci A, Cavallo MG, Purrello F, Anello M, Rotella CM, Bardini G, Vaccaro O, Riccardi G, Travaglini P, Morenghi E, Bosi E \& Pozzilli P. Clinical phenotype and beta-cell autoimmunity in Italian patients with adult-onset diabetes. European Journal of Endocrinology 2006154 441-447. (doi:10.1530/eje.1.02115)

8 Buzzetti R, Di Pietro S, Giaccari A, Petrone A, Locatelli M, Suraci C, Capizzi M, Arpi ML, Bazzigaluppi E, Dotta F, Bosi E \& The Non Insulin Requiring Autoimmune Diabetes (NIRAD) Study Group. High titer of autoantibodies to GAD identifies a specific phenotype of adult-onset autoimmune diabetes. Diabetes Care 200730 932-938. (doi:10.2337/dc06-1696)

9 Desai M, Cull CA, Horton VA, Christie MR, Bonifacio E, Lampasona V, Bingley PJ, Levy JC, Mackay IR, Zimmet P, Holman RR \& Clark A. GAD autoantibodies and epitope reactivities persist after diagnosis in latent autoimmune diabetes in adults but do not predict disease progression: UKPDS 77. Diabetologia 200750 2052-2060. (doi:10.1007/s00125-0070745-6)

10 Yang L, Zhou ZG, Huang G, Ouyang LL, Li X \& Yan X. Six-year follow-up of pancreatic beta cell function in adults with latent autoimmune diabetes. World Journal of Gastroenterology 200511 2900-2905.

11 Gottsäter A, Landin-Olsson M, Fernlund P, Lernmark A \& Sundkvist G. Beta-cell function in relation to islet cell antibodies during the first $3 \mathrm{yr}$ after clinical diagnosis of diabetes in type II diabetic patients. Diabetes Care 199316 902-910. (doi:10.2337/ diacare.16.6.902)

12 Falorni A, Gambelunghe G, Forini F, Kassi G, Cosentino A, Candeloro P, Bolli GB, Brunetti P \& Calcinaro F. Autoantibody recognition of $\mathrm{COOH}$-terminal epitopes of GAD65 marks the risk for insulin requirement in adult-onset diabetes mellitus. Journal of Clinical Endocrinology and Metabolism 200085 309-316. (doi:10. $1210 /$ jc.85.1.309)

13 Lohmann T, Kellner K, Verlohren HJ, Krug J, Steindorf J, Scherbaum WA \& Seissler J. Titre and combination of ICA and autoantibodies to glutamic acid decarboxylase discriminate two clinically distinct types of latent autoimmune diabetes in adults (LADA). Diabetologia $2001 \quad \mathbf{4 4}$ 1005-1010. (doi:10.1007/ s001250100602)

14 Radtke MA, Midthjell K, Nilsen TI \& Grill V. Heterogeneity of patients with latent autoimmune diabetes in adults: linkage to autoimmunity is apparent only in those with perceived need for 
insulin treatment: results from the Nord-Trøndelag Health (HUNT) study. Diabetes Care 200932 245-250. (doi:10.2337/ dc08-1468)

15 Hawa MI, Thivolet C, Mauricio D, Alemanno I, Cipponeri E, Collier D, Hunter S, Buzzetti R, de Leiva A, Pozzilli P, Leslie RDG \& Action LADA Group. Metabolic syndrome and autoimmune diabetes: action LADA 3. Diabetes Care 200932 160-164. (doi:10.2337/dc08-1419)

16 Worldwide Definition of the Metabolic Syndrome. Available from: www.idf.org/webdata/docs/IDF_metasyndrome_definition.pdf.

17 Falorni A, Örtqvist E, Persson B \& Lernmark ̊̊. Radioimmunoassays for glutamic acid decarboxylase (GAD65) and GAD65 autoantibodies using ${ }^{35} \mathrm{~S}$ or ${ }^{3} \mathrm{H}$ recombinant human ligands. Journal of Immunological Methods 1995186 89-99. (doi:10.1016/ 0022-1759(95)00139-2)

18 Marcovina SM, Landin-Olsson M, Essen-Miller A, Palmer JP \& Lernmark A. Evaluation of a novel radioimmunoassay using ${ }^{125}$ I-labelled human recombinant GAD65 for the determination of glutamic acid decarboxylase (GAD65) autoantibodies. International Journal of Clinical and Laboratory Research $2000 \mathbf{3 0}$ 21-26. (doi:10.1007/s005990070029)

19 Motzo C, Contu D, Cordell HJ, Lampis R, Congia M, Marrosu G, Todd JA, Devoto M \& Cucca F. Heterogeneity in the magnitude of the insulin gene effect on HLA risk in type 1 diabetes. Diabetes 200453 3286-3291. (doi:10.2337/diabetes.53.12.3286)

20 Isomaa B, Almgren P, Henricsson M, Taskinen MR, Tuomi T, Groop L \& Sarelin L. Chronic complications in patients with slowly progressing autoimmune type 1 diabetes (LADA). Diabetes Care 199922 1347-1353. (doi:10.2337/diacare.22.8.1347)

21 Hosszufalusi N, Vatay A, Rajczy K, Prohaszka Z, Pozsonyi E, Horvath L, Grosz A, Gero L, Madacsy L, Romics L, Karadi I, Fust G \& Panczel P. Similar genetic features and different islet cell autoantibody pattern of latent autoimmune diabetes in adults (LADA) compared with adult-onset type 1 diabetes with rapid progression. Diabetes Care 200326 452-457. (doi:10.2337/ diacare.26.2.452)

22 van Deutekom AW, Heine RJ \& Simsek S. The islet autoantibody titres: their clinical relevance in latent autoimmune diabetes in adults (LADA) and the classification of diabetes mellitus. Diabetic Medicine 200825 117-125. (doi:10.1111/j.1464-5491.2007. 02316.x)

23 Knip M, Kukko M, Kulmala P, Veijola R, Simell O, Akerblom HK \& Ilonen J. Humoral beta-cell autoimmunity in relation to
HLA-defined disease susceptibility in preclinical and clinical type 1 diabetes. American Journal of Medical Genetics 2002115 48-54. (doi:10.1002/ajmg.10343)

24 Kasuga A, Maruyama T, Nakamoto S, Ozawa Y, Suzuki Y \& Saruta T. High-titer autoantibodies against glutamic acid decarboxylase plus autoantibodies against insulin and IA-2 predicts insulin requirement in adult diabetic patients. Journal of Autoimmunity 199912 131-135. (doi:10.1006/jaut.1998.0261)

25 Kobayashi T, Tanaka S, Shimada A \& Maruyama T. High titer of autoantibodies to GAD identifies a specific phenotype of adultonset autoimmune diabetes: response to Buzzetti et al. Diabetes Care 200730 e126. (doi:10.2337/dc07-1104)

26 Verge CF, Gianani R, Yu L, Pietropaolo M, Smith T, Jackson RA, Soeldner JS \& Eisenbarth GS. Late progression to diabetes and evidence for chronic beta-cell autoimmunity in identical twins of patients with type 1 diabetes. Diabetes $1995 \mathbf{4 4} 1176-1179$. (doi:10.2337/diabetes.44.10.1176)

27 Murao S, Kondo S, Ohashi J, Fujii Y, Shimizu I, Fujiyama M, Ohno K, Takada Y, Nakai K, Yamane Y, Osawa H \& Makino H. Anti-thyroid peroxidase antibody. IA-2 antibody, and fasting C-peptide levels predict beta cell failure in patients with latent autoimmune diabetes in adults (LADA), A 5-year follow-up of the Ehime study. Diabetes Research and Clinical Practice $2008 \mathbf{8 0}$ 114-121. (doi:10.1016/j.diabres.2008.01.024)

28 Tuomi T, Groop LC, Zimmet PZ, Rowley MJ, Knowles W \& Mackay IR. Antibodies to glutamic acid decarboxylase reveal latent autoimmune diabetes mellitus in adults with a noninsulindependent onset of disease. Diabetes $1993 \mathbf{4 2} 359-362$. (doi:10.2337/diabetes.42.2.359)

29 Lindholm E, Hallengren B \& Agardh CD. Gender differences in GAD antibody-positive diabetes mellitus in relation to age at onset, C-peptide and other endocrine autoimmune diseases. Diabetes/ Metabolism Research and Reviews 200420 158-164. (doi:10. 1002/dmrr.420)

30 Bonifacio E, Mayr A, Knopff A \& Ziegler AG. Endocrine autoimmunity in families with type 1 diabetes: frequent appearance of thyroid autoimmunity during late childhood and adolescence. Diabetologia 200952 185-192. (doi:10.1007/ s00125-008-1206-6)

Received 7 June 2010

Accepted 5 July 2010 